\title{
Efficacy and Safety of Argon Laser Trabeculoplasty in Lowering Intraocular Pressure as Adjunctive Treatment to Primary Open Angle Glaucoma
}

\author{
Uzma Fasih, Erum Shahid, Arshad Sheikh
}

Pak J Ophthalmol 2019, Vol. 35, No. 1

See end of article for authors affiliations

Correspondence to:

Uzma Fasih

FCPS, Associate Professor

Eye department, Karachi Medical and Dental College Abbasi Shaheed Hospital Email:

yousufuzma@hotmail.com
Purpose: To assess efficacy and safety of argon laser trabeculoplasty (ALT) to lower intra ocular pressure (IOP) as adjunctive treatment in primary open angle glaucoma (POAG) patients.

Study Design: Descriptive Cross Sectional.

Place and Duration of Study: Ophthalmology Department, Abbassi Shaheed Hospital from May 2016 to August, 2017.

Materials and Methods: Primary open glaucoma patients were selected from eye OPD. Patients with Inflammatory, angle closure, traumatic glaucoma were excluded. Pre laser and Post laser IOP was measured at $1^{\text {st }}$ week then at 1,3 and 6 months. Data was collected and analyzed on SPSS version 20. There were 114 patients. Sample size was calculated by Rao Soft Sample size calculator.

Results: There were 69 (61\%) male patients. Mean age of patients was $60.53 \pm$ 10.71 years. Mean pre laser Intraocular pressure was $23.98 \pm 10.01 \mathrm{~mm} \mathrm{Hg}$. Mean post laser IOP at one month was $15.6 \pm 3.25$ and at 6 months was $14.8 \pm$ $3.28 \mathrm{~mm} \mathrm{Hg}$. Overall mean reduction of IOP was $9.18 \mathrm{~mm} \mathrm{Hg}$ from baseline, with $p$ value less than $<0.005$. Pre laser 3 medications were used by $20(18 \%)$ and 2 medications by 49 (43\%). At 6 months follow-up 4 (3.5\%) patients were on 3 medications and $31(37 \%)$ were on 2 medications. It has a p value $<0.005$. Drug free patients at 6 months follow-up were 37 (33\%). Mild iritis was seen in 7 (6.14\%) patients.

Conclusion: Argon Laser Trabeculoplasty is effective and safe method to control IOP in POAG when used as an adjunct to medical therapy. It also decreases number of drugs used by patients.

Key Words: Argon laser trabeculoplasty, intraocular pressure, primary open angle glaucoma.

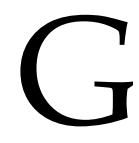
laucoma is the leading cause of irreversible blindness world over. It is estimated that 64 million people suffer from this diease around the world ${ }^{1}$. It is characterized by optic nerve damage, cupping of the optic disc and typical visual field defects. Rise in intra ocular pressure is a major risk factor. Reduction of intraocular pressure (IOP) may be helpful in reducing the optic nerve damage ${ }^{2}$. It is the third leading cause of irreversible blindness over the age of 40 years in Pakistan ${ }^{3}$.

Primary open angle glaucoma (POAG) commonly presents in our clinics where the cause of raised 
intraocular pressure is functional impairment of trabecular meshwork (TM). Medical treatment is the first line of treatment. Surgical treatment is generally offered when patient does not respond to medical treatment. Wise and Witter are credited for describing first successful protocol, which is widely known as Laser Trabeculoplasty (LTP) in $1979^{4}$.

The utilized energy source for modern LTP is continuous-wave argon laser, operated in the bluegreen wavelength spectrum $(454-529 \mathrm{~nm})^{5}$. The exact mechanism of the procedure is not known, but it is believed that the laser energy applied to the trabecular meshwork initiates structural and/or physiologic changes that facilitate aqueous outflow ${ }^{6}$.

Wise and Witter stated that thermal energy produced by pigment absorption of laser light causes fibrosis of collagen in trabecular lamellae. Subsequent shortening of treated meshwork might enlarge existing spaces between two treatment sites and expands Schlemm's canal by pulling meshwork centrally which facilitates the aqueous drainage from the trabecular meshwork ${ }^{4,7}$.

Microscopic and immune histochemical analysis of the trabecular meshwork after ALT has shown local photocoagulation of treated trabecular tissue, while cells adjacent to treated areas have shown increased phagocytic activity.

Moreover studies have reported changes in the extracellular matrix after LTP related to an induction of matrix metalloproteinases. These enzymes normally break down extracellular matrix to maintain normal turnover of trabecular meshwork. Thus there is cellular activation of trabecular mesh work with increased trabecular cell replication and increased number of cells involved in maintaining the outflow from trabecular meshwork ${ }^{8}$.

Laser trabeculoplasty (LTP) is a well known therapeutic method for the management of glaucoma since the last 30 years, which became popular during the past 10 years. LTP is an effective therapeutic option, as it can supplement topical medical treatment. One laser session can reduce the Intraocular pressure (IOP) for many years, thus reducing the adverse effects of poor compliance associated with medical treatment. More over LTP is in general a well tolerated and usually a low-risk procedure, as compared with glaucoma surgery ${ }^{5}$.

Majority of patients coming to government hospitals belong to poor socioeconomic class with poor compliance. This procedure will be safe and cost effective in lowering intraocular pressure. It will also decrease number of topical anti glaucoma medication which are a financial burden for them. Little work has been published on this topic in local journals. We conducted this study to asses efficacy and safety of argon laser trabeculoplasty to lower intra ocular pressure as adjunctive treatment in patients with primary open angle glaucoma.

\section{MATERIALS AND METHODS}

The study was carried out at ophthalmology department Abbasi Shaheed Hospital and Karachi Medical \& Dental College from May 2016 to August 2017. It was a descriptive cross sectional study. It was started after approval from Ethical Review Committee of the hospital. Informed consent was taken. Patients were registered through non probability consecutive sampling technique. Sample size was calculated using Rao Soft Sample Size Calculator taking confidence interval $90 \%$, Margin of error $5 \%$, population size 10,000 and prevalence $P$ for reduction in intraocular pressure $12.1 \mathrm{~mm} \mathrm{Hg}$ from the reference study. 9 Calculated sample size was 114 .

Patients 45 years of age or above, diagnosed with Primary open angle glaucoma with failed medical treatment were included in the study. Patients with angle closure glaucoma, inflammatory glaucoma, traumatic glaucoma, aphakic and pseudophakic glaucoma and those having history of previous trabeculectomy and cataract surgery were excluded from the study.

Written informed consent was taken from them. Detail history was taken. Ocular examination was conducted including visual acuity, slit lamp examination, intraocular pressure, gonioscopy and fundoscopy. Diagnosis of Primary open glaucoma was established after ocular examination, optical coherence tomography and visual fields.

These patients underwent laser trabeculoplasty under topical anaesthesia. Pressure lowering agents Brimonidine and Pilocarpine were instilled 1 hour before surgery. A Goldmann 3-mirror goniolens was placed on the eye with $1 \%$ methylcellulose. The helium-neon aiming beam was focused at the junction of pigmented and non pigmented trabecular meshwork with a spot size of 50 microns and duration of 0.1 seconds, keeping the starting energy level at 200 watts and increased according to the level of TM pigmentation. Blanching of the TM or bubble formation was taken as the end result. Approximately 
50 adjacent laser spots were applied over inferior $180^{\circ}$ of the TM, 25 spots in each quadrant.

Post laser IOP was measured at one hour, one day, one week, and one, three, and six months. These responses were recorded on predesigned performa. During the post laser follow-up period, patients were treated with mild steroid (flouromethalone) 4 times a day which was withdrawn after I week, topical nonsteroidal anti-inflammatory drug (nepafenac) 3 times a day which was continued for 1 month and topical antiglaucoma medications as required. Progression of glaucoma was monitored by visual fields and OCT (Optical coherence tomography) throughout the study.

Data was collected and analyzed on SPSS version 20. Means were computed for numerical variables like age, pre-laser intraocular pressure and post laser intraocular pressure with standard deviations. Frequencies of gender, eye, systemic diseases, visual acuity, prelaser and post laser medications were calculated. A paired $t$ test was used for assessing changes in IOP at different follow up visits. Reduction in number of topical medications pre laser and post laser treatment was assessed with help of pair $t$ test. $P$ value of less than 0.05 was taken as statistically significant.

\section{RESULTS}

A total of 114 patients were included in our study. Out of these male patients were (69) $61 \%$. Mean age of the patients was $60.53 \pm 10.71$ SD years ranging from 45 to 82 years. Right eye was treated in $54(47 \%)$ patients and left eye was treated in $60(53 \%)$ patients. All other demographic features of patients are given in Table 1. Mean pre laser IOP was $23.98 \pm 10.01 \mathrm{SD} \mathrm{mm} \mathrm{Hg}$. Mean IOP at day one post laser was $15.7 \pm 3.68 \mathrm{SD} \mathrm{mm}$ $\mathrm{Hg}$. Mean IOP at one month post laser follow up was $15.6 \pm 3.25 \mathrm{SD} \mathrm{mm} \mathrm{Hg}$. Mean IOP at 3 and 6 months post laser follow up visit was $14.8 \pm 2.61 \mathrm{SD} \mathrm{mm} \mathrm{Hg}$ and $14.8 \pm 3.28 \mathrm{SD} \mathrm{mm} \mathrm{Hg}$ respectively. There was an overall mean reduction of $9.18 \mathrm{~mm} \mathrm{Hg}$ from baseline which was statistically significant (Table 2). Pre laser patients on 3 medications were $20(18 \%)$ and those on 2 medications were $49(43 \%)$ while at 3 months follow $4(3.5 \%)$ patients were on 3 medications and $39(34 \%)$ were on 2 medications. At 6 months follow-up 4 (3.5\%) patients were on 3 medications and 31 (37\%) patients were on 2 medications (Table 3 ). This reduction in medications was statistically significant with $\mathrm{p}$ value $<$ 0.005. Drug free patients at 6 months follow up were $37 \quad(33 \%)$ (Table 1). No significant post laser complications were seen except mild iritis in 7 (6.14\%) patients and transient rise in IOP in $2(1.75 \%)$ patients which were managed with topical steroids and pressure lowering agents.

Pair T test was conducted to evaluate the effect of Argon laser trabeculoplasty on intra ocular pressure. There was a significant difference in mean pre laser IOP (23.98 $\pm 10.01 \mathrm{~mm} \mathrm{Hg} \mathrm{SD)}$ and 1 month post laser IOP $(15.5 \pm 3.2 \mathrm{~mm} \mathrm{Hg} \mathrm{SD})$ with $95 \% \mathrm{CI}$ ranging from 6.66 to 10.1 with $\mathrm{p}$ value of less than 0.005 . Three

Table 1: Demographic Characteristics.

\begin{tabular}{|lcc|}
\hline Variables & Frequencies & Percentages \\
\hline Mean Age in years & $60.53 \pm 10.71 S D$ & \\
Minimum & 45 & \\
maximum & 82 & $61 \%$ \\
Gender & 69 & $47 \%$ \\
Male & 54 & $53 \%$ \\
Right eye & 60 & \\
Left eye & $6 \pm 3.6$ years & $18.4 \%$ \\
Duration of glaucoma (mean) years & & $16 \%$ \\
Visual acuity & 21 & $36 \%$ \\
$6 / 6-6 / 9$ & 18 & $30 \%$ \\
$6 / 12-6 / 18$ & 41 & $13 \%$ \\
$6 / 24-6 / 60$ & 34 & $6 \%$ \\
Less than $6 / 60$ & & $68.4 \%$ \\
Systemic disease & 15 & $33 \%$ \\
HTN & 7 & \\
DM & 78 & \\
Both eyes & 37 & \\
Drug free at 6 months post laser & & \\
\end{tabular}


Table 2: Post Alt Reduction in IOP.

\begin{tabular}{|clccc|}
\hline No. & IOP & Mean IOP \pm SD mmhg & Comparison & P value \\
\hline 1. & Pre laser & $23.98 \pm 10.01$ & & \\
2. & 1 day & $15.7 \pm 3.68$ & $1 \& 2$ & $<.000$ \\
3. & 1 month & $15.6 \pm 3.25$ & $1 \& 3$ & $<.000$ \\
4. & 3 months & $14.8 \pm 2.61$ & $1 \& 4$ & $<.000$ \\
5. & 6 months & $14.8 \pm 3.28$ & $1 \& 5$ & $<.000$ \\
\hline
\end{tabular}

Table 3: Post Alt Reduction in Medications.

\begin{tabular}{|lcc|}
\hline & Frequency & $\%$ \\
\cline { 1 - 1 } Pre laser medication & & \\
1 medication & 25 & $22 \%$ \\
2 medications & 49 & $43 \%$ \\
3 medications & 20 & $18 \%$ \\
4 medications & 20 & $18 \%$ \\
Post laser 3 month & & \\
1 medication & 45 & $40 \%$ \\
2 medications & 39 & $34 \%$ \\
3 medications & 4 & $3.5 \%$ \\
4 medications & 0 & \\
Post laser 6 month & 38 & $33 \%$ \\
1 medications & 35 & $31 \%$ \\
2 medications & 4 & $3.5 \%$ \\
3 medications & 0 & \\
4 medications & & \\
\hline
\end{tabular}

Table 4: Pair-T Test.

\begin{tabular}{|cll|}
\hline No. & No of Medications & P value \\
\hline 1. & Prelaser medications \& at 3 month & $<.000$ \\
2. & Prelaser medication \& at 6 month & $<.000$ \\
\hline
\end{tabular}

month post laser mean IOP was $14.8 \pm 2.61 \mathrm{SD} \mathrm{mm} \mathrm{Hg}$ with $95 \%$ CI ranging from 7.4 to 10.8 and $\mathrm{P}$ value of less than .005. Six month post laser mean IOP was 14.8 $\pm 3.2 \mathrm{~mm} \mathrm{Hg}$ SD with $95 \% \mathrm{CI}$ ranging from 7.4 to 10.9 and $\mathrm{p}$ value of less than .005 . So the difference between pre laser mean IOP and post laser mean IOP at 1,3 and 6 months was highly significant.

Pair $\mathrm{T}$ test was conducted to see the reduction in prelaser and post laser medication. Mean difference in prelaser medication was 2.307 with $95 \%$ confidence interval from 2.12 to 2.49 . While 6 months post laser medication mean difference was 1.053 with $95 \%$ confidence interval ranging from 0.89 to 1.22 . with $\mathrm{p}$ value less than 0.005 which was highly significant.

\section{DISCUSSION}

In the developing world treatment options for glaucoma are becoming more complicated as all treatment modalities and health care facilities are not available in all environments. In addition cost of medicines and hurdles in their distribution make medical treatment of glaucoma very difficult and sometimes impossible in most of the developing countries. Prostaglandin analogs are said to be the first line of treatment currently ${ }^{10}$. Topical B blockers, alpha adrenergic agonists and Carbonic anhydrase inhibitors may be used as additional therapies when required but there may be a significant inter patient variability in their response.11,12.

ALT has been known to be an effective method for reduction of IOP in POAG patients. Use of this modality as an adjunctive to the medical treatment cannot be overlooked. ALT does not cure glaucoma but provides a long term reduction in IOP when used as an adjunctive treatment to medical treatment of glaucoma. In addition ALT cannot be relied upon as primary treatment option in patients who have poor compliance as there may be long periods of uncontrolled IOP. So ALT could be a better adjunctive treatment for glaucoma rather than primary treatment ${ }^{10}$.

We conducted this study to document the efficacy of this procedure as an adjunctive therapy to medical treatment in patients of POAG. We found in our study that there was a mean reduction of $9.18 \mathrm{~mm} \mathrm{Hg}$ in post laser IOP as compared to base line and 6 month follow up period there was a significant reduction in number of topical medications. A randomized clinical trial known as Glaucoma Laser Trial GLT was carried out in America to assess safety and efficacy of LTP for controlling IOP in POAG patients .They reported a mean reduction of $9 \mathrm{~mm} \mathrm{Hg}$ in IOP among the laser treated eyes as compared to $7 \mathrm{~mm} \mathrm{Hg}$ reduction in IOP among those who were treated with medical treatment. The study also reported that ALT could be regarded as an equally effective therapy as medical treatment for POAG patients. Our study also reported similar reduction in IOP'2.

Shawartz and Coworkers also reported a drop of $9.7 \mathrm{~mm} \mathrm{Hg}$ in mean IOP at 2 months follow-up, 7.3 
$\mathrm{mm} \mathrm{Hg}$ at 2 years and $4.9 \mathrm{~mm} \mathrm{Hg}$ at 5 years follow up after Argon Laser Trabeculoplasty in cases of POAG ${ }^{13}$. Speath and Et al concluded in their study that LTP deferred the need of surgical treatment in about one third of the patients of POAG ${ }^{14}$.

Sharma and Gupta reported $76 \%$ success rate of ALT in POAG in their study. ${ }^{9}$ One of the local study by Mehar and et al reported $12.1 \mathrm{mmHg}$ reduction in mean IOP at 6 months follow up in their case series of 35 eyes $^{15}$.

Detry-Morel et al also reported a mean reduction of $4.9 \mathrm{mmhg}$ in IOP and significant reduction in number of drugs required at final visit along with minimal anterior chamber inflammation ${ }^{16}$. Grinich et al reported $7.8 \mathrm{mmmHg}$ reduction in IOP at 3 years follow-up ${ }^{17}$. Achadi Et al reported $40.3 \%$ success rate of ALT at 5 years follow up ${ }^{18}$.

Agarwal and et al reported reduction of $7.2 \mathrm{~mm}$ $\mathrm{Hg}$ in mean IOP at 6 month follow up after LTP which is quite similar to $9.18 \mathrm{~mm} \mathrm{Hg}$ post laser reduction of IOP in our study. Post Laser complications in their study like transient rise of IOP was found to be $51.3 \%$ patients, Iritis in 5\% patients, formation of peripheral anterior synechia in $2.5 \%$ patients and rarely hyphaema ${ }^{19}$.

The frequency of complications was fewer in our study like mild iritis was seen in $7(6.14 \%)$ patients, transient rise in IOP in $2(1.75 \%)$ patients and no hyphaema. The reason for few complications could be that patients were kept on topical nonsteroidal antinfammatory drugs in addition to steroids and topical brimonidine.

In our study pre laser patients on 3 medications were $20(18 \%)$, those on 2 medications were $49(43 \%)$ and on one medication were $25(22 \%)$. At 6 months follow up $4(3.5 \%)$ patients were on 3 medications and $35(31 \%)$ patients were on 2 medications and 38(33\%) on one medication. This reduction in medications was statistically significant $\mathrm{p}$ value $<0.005$. Drug free patients at 6 months follow up were $37(33 \%)$ while 4 $(3.7 \%)$ patients had to be supplemented with trabeculectomy due to uncontrolled IOP at 6 months follow up. In study by Agarwal 10.2\% patients underwent trabeculectomy at 6 momths follow up. Agarwal and et al reported $10 \%$ drug free patients, $15 \%$ on one medication and $20 \%$ on 2 medications at 5 year follow up. The differences from our study could be due to their prolonged follow up period ${ }^{19}$.

Adequate safety and efficacy profiles of laser trabeculoplasty have been reported Due to the reliable safety and efficacy of laser trabeculoplasty this procedure can be used at any time during the treatment of POAG as primary or adjunctive therapy and also decreases the need of any medical treatment before going for any surgical intervention ${ }^{20}$.

It can also be tried before cataract surgery of glaucoma patients to achieve a better management of IOP and to reduce the number of medications in patients who are on more than one drug. In addition it can be used in patients who are at risk of rise in IOP after receiving intravitreal injections ${ }^{21}$. Majority of the studies reported $25-30 \%$ post laser decrease in IOP22.

Another study reported that if LTP is used earlier in treatment of POAG the risk of adverse effect is diminished as the number of medications are decreased to reduce the IOP. ${ }^{23}$ Stein Et al reported that LTP is economical and cost effective as compared to medical treatment ${ }^{24}$.

Limitation of our study is short term follow up period. Long term follow up is required to determine efficacy of ALT as an adjunctive therapy for prolonged control of IOP in POAG.

\section{CONCLUSION}

We concluded from our study that ALT is a safe and effective method to control IOP in POAG when used adjunct to medical therapy. It helps in decreasing the number of topical medications used for management of POAG and is thus cost effective.

\section{Conflict of Interest: None.}

\section{Author's Affiliation}

Dr. Uzma Fasih

FCPS, Associate Professor

Eye department, Karachi medical and dental college Abbasi Shaheed Hospital.

Dr. Erum Shahid

MCPS, FCPS, Assistant professor

Eye department, Karachi medical and dental college Abbasi shaheed hospital

Dr. Arshad Sheikh

MCPS, FCPS, professor and Head of department

Eye department, Karachi medical and dental college Abbasi shaheed hospital 


\section{Role of Authors}

Dr. Uzma Fasih

Concept design, data collection, Manuscript writing,

Data analysis, Critical review.

Dr. Erum Shahid

Concept, Design, critical review.

Dr. Arshad Sheikh

Concept design, data collection, Critical review.

\section{REFRENCES}

1. Tham YC, Li X, Wong TY, Quigley HA, Aung T, Cheng CY. Global prevalence of glaucoma and projections of glaucoma burden through 2040: a systematic review and meta-analysis. Ophthalmology, 2014; 121 (11): 2081-2090.

2. Sommer A. Introcular Pressure and Glaucoma Am J Ophthalmol. 1989; 107: 186-8.

3. Dineen B, Bourne RR, Jadoon Z, et al. Causes of blindness and visual impairment in Pakistan. The Pakistan national blindness and visual impairment survey. Br J Ophthalmol. 2007;91(8):1005-10.

4. Wise JB, Witter SL. Argon laser therapy for open-angle glaucoma. A pilot study. Arch Ophthalmol. 1979; 97 (2): 319-322.

5. The Glaucoma Laser Trial Research Group.The Glaucoma Laser Trial (GLT). 2. Results of argon laser trabeculoplasty versus topical medicines. Ophthalmology. 1990; 97 (11): 1403-1413.

6. Stein JD, Challa P. Mechanisms of action and efficacy of argon laser trabeculoplasty and selective laser trabeculoplasty. Curr Opin Ophthalmol. 2007; 18 (2): 140-145.

7. Bradly JM, Anderssohn AM, Clovis CM, et al. Mediation of Laser Trabeculoplasty induced matrix metlloprotienase expression by IL-beta and TNF alpha. Invest Ophthalmol Vi Sci. 2000; 41: 422.

8. Singh K, Shrivastava A. Medical management of glaucoma: Principles and practice Indian J Ophthalmol. 2011 Jan; (1): S88-S92.

9. Sharma A, Gupta A. Primary Argon Laser Trabeculoplasty Vs Pilocarpine in Primary Open angle glaucoma 2 years follow up study Br J Ophthalmol. 1997; 45: 109-13.

10. Feldman RM, Tanna AP, Gross RL, Chuang AZ, Baker L, Reynolds A, Prager TC. Additivity Study Group. Comparison of the Ocular Hypotensive Efficacy of Adjunctive Brimonidine $0.15 \%$ or Brinzolamide $1 \%$ in Combination with Travoprost $0.004 \%$ Ophthalmology, 2007; 114: 1248-54.

11. Bagga H, Liu JH, Weinreb RN. Intraocular pressure measurements throughout the $24 \mathrm{~h}$. Curr Opin Ophthalmol. 2009; 20: 79-83. [PubMed]

12. The Glaucoma Laser Trial (GLT) 2, Results of Argon Laser Trabeculoplasty Vs Topical Medication. The Glaucoma Research Group. Ophthalmology, 1990 ; 97: 1403-13.

13. Shcawarz AL, Love DG, Schawartz MA. Long Term follow up of Argon Laser Trabeculoplasty for un controlled Open Angle Glaucoma Arch Ophthalmol. 1985; 103: 1482-4.

14. Speath GL, Baez KA. Argon Laser Trabeculoplasty controls one third of cases of Progressive uncontrolled Open Angle Glaucoma for 5 years Arch Ophthalmol. 1999; 110: 491-4.

15. Mehar P.S, Jalai KK. Argon Laser Trabeculoplasty as Primary therapy in Primary Open Angle Glaucoma JCPSP. 2008; 18 (2): 102-104.

16. Detry-Morel M, Muschart F, Pourjavan S. Micropulse diode laser $(810 \mathrm{~nm})$ versus argon laser trabeculoplasty in the treatment of open-angle glaucoma: comparative short-term safety and efficacy profile. Bull Soc Belge Ophthalmol. 2008; 308: 21-28.

17. Grinich NP, Buskirk MV, Samples JR. Three year efficacy of argon laser trabeculoplasty. Ophthalmology, 1987; 94: 858-62.

18. Adachi M, Shirato S, Kaburagi T, et al. Ten year results of argon laser trabeculoplasty. Nippon Ganka Gakkai Zasshi. 1994; 98: 374-8.

19. Agarwal HC, Sihota R, Das C, Dada T. Role of Argon Laser Trabeculoplasty in Primary Open angle Glaucoma in Indian patients Br J Ophthalmol. 2002; 86: 733-6.

20. Mansouri K, Shaarawy T. Comparing pattern scanning laser trabeculoplasty to selective laser trabeculoplasty: a randomized controlled trial Acta Ophthalmol. 2017; 95: 361-365.

21. Bozkurt E, Kara N. Yazici AT, et al. Prophylactic selective laser trabeculoplasty in the prevention of intraocular pressure elevated after intravitreal triamcinolone acetonide injection. Am J. Ophthlamol. 2011; 152: 976-981.

22. Latina MA, Sibayan SA, Shin DH, et al. Q switched $532 \mathrm{~nm} \mathrm{Nd}$ : YAG laser trabeculoplasty (selective laser trabeculoplasty): a multicenter, pilot clinical study. Ophthalmology, 1998; 105: 2082-2088, Discussion, 20892090.

23. Francis BA, Ianchulev T, Schofield JK, Minckler DS. Selective laser trabeculoplasty as a replacement for medical therapy in open-angle glaucoma. Am J Ophthalmol. 2005; 140 (3): 524-525.

24. Stein JD, Kim DD, Peck WW, et al. Cost-effectiveness of medications compared with laser trabeculoplasty in patients with newly diagnosed open-angle glaucoma. Arch Ophthalmol. 2012; 130 (4): 497-505. 